\title{
piRNA/ PIWI protein complex as potential biomarkers in sporadic amyotrophic lateral sclerosis
}

Rehab F. Abdelhamid ( $\square$ rehab.abdelhamid@gmail.com )

Osaka University Graduate School of Medicine. https://orcid.org/0000-0001-9351-3371

\section{Kotaro Ogawa}

Department of Neurology, Osaka University Graduate school of Medicine

\section{Goichi Beck}

Department of Neurology, Osaka University, Graduate School of Medicine: Osaka Daigaku Daigakuin Igakukei Kenkyuka Igakubu

\section{Kensuke Ikenaka}

Department of Neurology, Osaka University graduate School of Medicine

\section{Eriko Takeuchi}

Osaka University School of Medicine Graduate School of Medicine: Osaka Daigaku Daigakuin Igakukei Kenkyuka Igakubu

\section{Yoshiaki Yasumizu}

Osaka University

Jyunki Jinno

Department of Neurology; Osaka University Graduate School of Medicine

\section{Yasuyoshi Kimura}

Neurology Depertment Osaka University Graduate School of Medicine

\section{Kousuke Baba}

Department of Neurology, Osaka University Graduate School of Medicine

\section{Yoshitaka Nagai}

Kindai University: Kinki Daigaku

\section{Yukinori Okada}

Osaka University Graduate School of medicine

\section{Yuko Saito}

Tokyo Metropolitan University

\section{Shigeo Murayama}

Osaka University: Osaka Daigaku

\section{Hideki Mochizuki}

Department of Neurology Osaka University Graduate School of Medicine

\section{Seiichi Nagano}


Department of Neurology, Osaka University School of Medicine Graduate School of Medicine: Osaka Daigaku Daigakuin Igakukei Kenkyuka Igakubu

\section{Research Article}

Keywords: amyotrophic lateral sclerosis, miRNA, piRNA, PIWI protein, TDP-43

Posted Date: August 26th, 2021

DOl: https://doi.org/10.21203/rs.3.rs-843093/v1

License: (c) (1) This work is licensed under a Creative Commons Attribution 4.0 International License. Read Full License

Version of Record: A version of this preprint was published at Molecular Neurobiology on January 11th, 2022. See the published version at https://doi.org/10.1007/s12035-021-02686-2. 


\section{Abstract}

The pathological hallmark in the majority of amyotrophic lateral sclerosis (ALS) cases is the mislocalization and aggregation of TAR DNA-binding protein 43 (TDP-43), an RNA-binding protein. Several studies have attributed disease processes of ALS to abnormal RNA metabolism. However, dysregulated biogenesis of RNA, especially non-coding RNA (ncRNA), is poorly understood. To resolve it, RNA-Seq, biochemical and immunohistochemical analyses were performed on sporadic ALS (sALS) and control postmortem brain samples. Here we report perturbation of ncRNA biogenesis in PIWl-interacting RNA (piRNA) in several sALS brain samples associated with TDP-43 pathology. In addition, we confirmed the dysregulation of two PIWI homologs, PIWI-like-mediated gene silencing 1 (PIWIL1) and PIWIL4, which bind to piRNAs to regulate their expression. PIWIL1 was mislocalized and co-localized with TDP-43 in motor neurons of sporadic ALS lumbar cords. Our results imply that dysregulation of piRNA, PIWIL1, and PIWIL4 is linked to pathogenesis of ALS. Based on these results, piRNAs and PIWI proteins are expected to be potential diagnostic biomarkers and therapeutic targets of ALS.

\section{Introduction}

During the last decade, significant progress has been made in identifying genes $(\sim 50)$ responsible for familial amyotrophic lateral sclerosis (fALS)[1]. However, fALS constitutes only $10 \%$ of all ALS cases. The remaining $90 \%$ are termed "sporadic" ALS, and most of these cases have no known genetically inherited component. The lack of an identifiable cause of these sporadic cases makes it difficult to diagnose ALS early enough to distinguish it from other diseases causing similar symptoms.

TAR DNA-binding protein 43 (TDP-43) is a highly conserved and ubiquitously expressed RNA-binding protein, which belongs to the heterogeneous nuclear ribonucleoprotein (hnRNP) family[2]. One of the hallmarks of ALS in the vast majority of both familial and sporadic ALS patients is mislocalization, aggregation, and inclusion formation of TDP-43 protein in brain tissues. The function of TDP-43 strongly suggests its role in RNA metabolism of coding and non-coding RNAs (ncRNAs), including small ncRNAs. Apart from fALS, the only possible way to study the pathogenesis of sporadic ALS (sALS) is to compare patient samples to control samples in order to discover potential gene regulatory targets, which should be beneficial for establishing biomarkers to diagnose the disease and to develop treatment strategies to block their aberrant expression.

Based on these findings, we conducted a pilot RNA-seq study using postmortem brain samples of sALS patients and controls with no known neurological disease (Fig. 1). Differential expression analysis revealed dysregulation in small ncRNA, particularly in micro RNA (miRNA) and PIWl-interacting RNA (piRNA). Owing to recent reports of potential involvement of piRNAs in the etiology of different neurological diseases, such as Rett syndrome, Alzheimer's disease (AD), Parkinson's disease (PD) [3], and brain aging [4], we focused on piRNA dysregulation in sALS samples to identify targets of those piRNAs and to determine whether PIWI protein/ piRNAs might contribute to ALS pathology. 


\section{Methods}

\section{Postmortem brain samples}

Postmortem human tissue samples used in this study were collected from the Brain Bank for Aging Research (BBAR) in the Tokyo Metropolitan Geriatric Hospital and Institute of Gerontology (TMGHIG), and the National Center of Neurology and Psychiatry (NCNP) Brain Bank. sALS cases $(n=7)$ were diagnosed based on El Escorial and Airlie House revised criteria with no familial history of the disease, and confirmed pathological inclusions of TDP-43 in brain lesions. Age- and sex-matched control cases $(n=7)$ had no neurological pathologies (Table 1). The pyramidal tract of the medulla oblongata was chosen for this study because this area is rich in axons of upper motor neurons, which are affected in ALS.

\section{cDNA preparation for small RNA-Seq}

Total RNA used for RNA-seq was extracted using Isogen-LS (Nippon Gene \# 311-02501). RNA concentration was measured with a Qubit RNA Assay Kit in a Qubit 2.0 Fluorometer (Life Technologies, CA, USA). RNA integrity was evaluated with RNA Pico 6000 Assay Kit and a Bioanalyzer 2100 System (Agilent Technologies, CA, USA). The mean RIN for ALS samples was 7.9 (range 6.9-8.5). (See Supplementary Information for raw values.) $50 \mathrm{ng}$ of each RNA sample were used to construct a cDNA library for each sample using a NEXTFLEX Combo-Seq mRNA/miRNA kit (Bio-Scientific), according to the manufacturer's protocol (Nova-5139-01). cDNAs were sequenced on an Illumina HiSeq 3000 with 74-bp single reads (10M reads/sample).

\section{RNA-Seq bioinformatic analysis}

We excluded adapter sequences using the cutadapt tool V.1.9.2[5]. We excluded reads shorter than 15 bases using trimmomatic version 0.36[6]. Then, we conducted comprehensive small RNA-seq analysis using the exceRpt extra-cellular RNA processing toolkit[7].

\section{Data Resources}

Raw FASTQ files for the RNA-seq libraries have been submitted to the DNA Data Bank of Japan (DDBJ) with Temporary Submission ID: SSUB016807.

\section{RNA extraction for reverse transcription-quantitative PCR (RT-qPCR) validation}

Total RNA was extracted from medulla oblongata samples using a mirVana kit (Ambion), according to manufacturer instructions. Small RNA (sRNA) was isolated from total RNA using the mirVana kit (Ambion), according to the manufacturer's instructions for SRNA isolation.

\section{RT-qPCR for target piRNA}

In brief, 3'-ends of $60 \mathrm{ng}$ of sRNA from each sample were ligated to 5' pre-adenylated 3'-adaptors (5'rApp/CTGTAGGCACCATCAAT/3ddC-3') using truncated T4RNA ligase 2 enzyme (NEB). 3'- adaptor-ligated 
sRNAs were reverse transcribed with ReverTraAce, reverse transcription polymerase (Toyobo) using an oligonucleotide complementary to the 3'-adaptor (IDT) in a MiniAmp plus Thermal Cycler (Applied Biosystems). RT-qPCR was performed using forward primers for each target piRNA and the oligonucleotide complementary to the 3 '-adaptor was used as a reverse primer (Table 5). Data were collected in triplicate for each sample on an ABI 7900 Prism qPCR machine and normalized using U6RNA as an internal control. Relative gene-expression levels were calculated using the fold-change method.

\section{RT-qPCR for coding genes}

RT-qPCR was performed using ReverTraAce (Toyobo), in a MiniAmp plus Thermal Cycler (Applied Biosystems). The glyceraldehyde-3-phosphate dehydrogenase gene (GAPDH) was used as an internal control to normalize coding genes. All quantitative PCR was performed using SYBRGreen qPCR Master Mix (Applied Biosystems) on an ABI 7900 Prism qPCR machine. Primer sets used for RT-qPCR are listed in Table 5.

\section{Standard curve method for RT-qPCR}

CDNA generated by reverse transcription for seven controls and seven ALS samples were used for the fold-change calculation. Equal volumes of control cDNA were pooled as a control stock. The control stock was then diluted 10 times with nuclease-free water in a dilution series to produce a control standard curve. SALS cDNA samples were diluted 20 times and then plotted against the control standard curve for each gene in triplicate. GAPDH was used as an internal control for normalization.

\section{$\triangle$ CT method for RT-qPCR}

The $\triangle$ CT RT-qPCR method was used to calculate the difference in cycle numbers needed to amplify target genes after normalization using the internal control. Therefore, $\triangle \mathrm{CT}$ denotes the $\mathrm{CT}$ value of a target gene substituted by the $\mathrm{CT}$ value of the internal control. Internal controls for piRNA and coding genes were U6RNA and GAPDH, respectively.

\section{Tissue lysates and protein quantification}

CellLytic MT Mammalian Tissue Lysis Reagent (C3228, Sigma-Aldrich), mixed with a protease inhibitor cocktail (1:100, EMD Millipore), was added to frozen tissue in 2-mL Lysing Matrix D tubes (P000912LYSKO, Precellys) to be homogenized using a MINILYS personal homogenizer (Bretin Instruments). Homogenized tissue lysates were centrifuged and supernatants were used for protein concentration determinations with a Pierce TM BCA protein assay (23225, Thermo Fisher Scientific).

\section{Immunoblotting for PIWI proteins}

$30 \mathrm{\mu g}$ of protein from lysates were separated on 5-20\% SDS-PAGE gels (E-T520L, ATTO) and transferred to 0.2- $\mu \mathrm{m}$ PVDF membranes (1620177, Bio-Rad). Membranes were blocked for $1 \mathrm{~h}$ in EZ blocking solution (AE-1475, ATTO) and then incubated with primary antibodies diluted in Can Get Signal Solution 1 (TOYOBO) overnight at $4^{\circ} \mathrm{C}$. Primary antibodies used included PIWIL1 (1:1000; \#701177, Novex), PIWIL2 
(1:500; ab181340, Abcam), PIWIL3 (1:500; ab77088, Abcam), PIWIL4 (1:1000; ab111714, Abcam), and PIWIL4 (1:1000; PA-49710, Thermo Fisher Scientific).

After primary antibody incubation, membranes were washed with $0.1 \%$ T-TBS buffer three times (for 15 , 10, 5 min each) before incubation with secondary antibody. Secondary antibodies were ECL anti-mouse antibody (1:10000; NA931V, GE Healthcare) or anti-rabbit antibody (1:20000; NA934V, GE Healthcare) diluted in Can get Signal solution2 (TOYOBO) or EZ block solution and supplemented with STREPTACTIN (\#1616380, Bio-Rad) for marker band detection. After secondary antibody incubation, membranes were washed with $0.1 \%$ T-TBS buffer $3 \times 10$ min before visualization.

\section{Data analysis and statistics (Immunoblotting and RT-qPCR)}

Statistics were performed using GraphPad Prism, version9 software. Two-way ANOVA was performed with Bonferroni's multiple comparison test to compare two or more independent groups. Pairwise comparisons were made using the Mann-Whitney test for $\triangle \mathrm{CT}$ with unpaired and nonparametric settings. Pairwise comparisons for fold change using the standard curve method were made using one-sample ttests (one-tailed Wilcoxon test) with paired and nonparametric settings.

For immunoblot statistical analysis, two-way ANOVA was performed using Bonferroni's multiple comparison test to compare two or more proteins. Pairwise comparisons were made using the MannWhitney test for PIWI proteins with unpaired and nonparametric, two-tailed p-value settings. p-values < 0.05 were considered significant ( $*$ indicates $p<0.05$ and $* * p<0.01$ ). All numbers in plots represent means \pm SEM.

\section{Neuropathological examination}

Clinical profiles of patients examined in the present study are shown in Supplementary Table 1. Samples from the lumbar cord (L5) were fixed in $10 \%$ buffered formalin. For immunohistochemistry (IHC), 6- $\mu \mathrm{m}$ sections were prepared. Deparaffinized sections were incubated 30 min with $0.3 \%$ hydrogen peroxide to quench endogenous peroxidase activity and then washed with PBS. The primary antibody was a mouse monoclonal antibody against PIWIL1 (1:500). Samples were autoclaved $15 \mathrm{~min}$ before incubation with antibody. Secondary antibody was goat anti-mouse immunoglobulin conjugated to peroxidase-labeled dextran polymer (Dako Envision+, Dako). Reaction products were visualized with 3,3'-diaminobenzidine tetrahydrochloride (ImmPACT DAB, Vector Laboratories), and hematoxylin was used to counterstain cell nuclei. For double IHC, two primary antibodies were combined, including antibodies against TDP-43 (1:1000, rabbit polyclonal, Proteintech) and PIWIL1 (1:500). Alexa Fluor® 488 goat anti-mouse $\lg \mathrm{G}(\mathrm{H}+\mathrm{L})$ antibody (A-11008, Thermo Fisher Scientific) and Alexa Fluor ${ }^{\circledR} 568$ goat anti-rabbit IgG $(\mathrm{H}+\mathrm{L})$ antibody (A-11004, Thermo Fisher Scientific) were used as secondary antibodies. Sudan Black B treatment was performed to reduce autofluorescence from lipofustin. Images were obtained using an all-in-one fluorescence microscope (BZ-X710, Keyence).

\section{Results}




\section{RNA-Seq analysis for sALS postmortem samples}

cDNA libraries were prepared using a Combo-Seq kit from seven control and seven sALS postmortem samples (Table 1). This kit was used to analyze poly A-tailed RNA and small ncRNA at the same time. However, it was difficult to get reliable annotation for poly A-tailed RNA during the bioinformatic analysis due to short reads, so we focused on analyzing only reads for small RNA-seq output. Data from two controls (CTR-1 and CTR-7) and SALS (ALS-1 and ALS-3) were excluded from the analysis due to low read numbers obtained from those libraries. Therefore, differential expression analysis was done using five controls and five sALS samples.

The analysis revealed significant dysregulation $(p<0.05)$ of nine miRNAs in ALS samples compared to controls (Fig. 2; Table 2). Among dysregulated miRNAs, hsa-miR-143-5p and hsa-miR-143-3p [8-10]were previously reported as dysregulated in cerebrospinal fluid (CSF), serum, and immortalized lymphoblast cell lines (LCLs) derived from ALS patient samples, validating parameters used for data analysis in this study.

\section{piRNA dysregulation in postmortem samples}

piRNAs are a class of small ncRNA molecules comprising 24-32 nucleotides, which protect genomic DNA mainly in germ cells by association with PIWI proteins, a subfamily of Argonaute proteins $[11,12]$. Five piRNAs were dysregulated in sALS samples (Fig. 3). Among them, three piRNAs were up-regulated (hsa-piR-000578, hsa-piR-020871, and hsa-piR-022184) and two piRNAs were down-regulated (hsa-piR009294 and hsa-piR-016735), using a false discovery rate (FDR) $<0.05$ and $p<0.05$ (Table 3).

There are no previous reports of these piRNAs in human brain. The piRNA sequence and target gene sequences were extracted from the PIWI-interacting RNA (piRNA) Database - piRNAdb [13] and predicted target genes for validation from piRBase [14] (Table 4). hsa-piR-33151, which was reported as decreased in serum samples from ALS patients [9], was not detected in our brain samples.

Dysregulation of the five piRNAs was validated using RT-qPCR with two methods, the standard curve method to visualize the overall trend for piRNA perturbation and the $\triangle \mathrm{CT}$ method to quantify expression in individual samples. RT-qPCR results confirmed the RNA-Seq analysis and these five piRNAs were significantly altered in sALS samples in comparison with controls (Fig. 4).

\section{Expression of piRNA target genes in postmortem samples}

The $\triangle \mathrm{CT}$ method was used to investigate the change in expression of in silico predicted potential target genes for dysregulated piRNA, according to piRBase and piRNAdb. Those genes are listed in Table 4 . Most target genes were ribosomal protein pseudogenes, RPL10P7, RPL13AP3, RPL18AP3, and 5S ribosomal RNA pseudogene transcripts, RNA5SP202 and RNA5-8SP6 (Fig. 5). Due to increased evidence of ribosomal protein dysregulation [15], we investigated expression of ribosomal protein coding genes instead of pseudogenes. There were no notable changes except for expression of RPL13A ( $p=0.053)$. Among expected target genes, no significant difference of TXNRD1 was observed between sALS and 
control samples. These data suggest that these piRNAs have regulatory effects beyond in silico-predicted target coding gene regulation.

\section{Dysregulation of PIWI proteins in postmortem samples}

piRNAs are produced by PIWI proteins from different genomic loci and function via the PIWI/ piRNA complex. Therefore, we investigated expression differences of PIWI proteins in postmortem samples using RT-qPCR and immunoblotting. PIWIL2 and PIWIL3 were not detected in immunoblots (data not shown) from all the samples. RT-qPCR using the $\triangle$ CT method for PIWIL2 and PIWIL3 showed insignificant differences in $\triangle \mathrm{CT}$ between sALS and control samples (Fig. 6A). PIWIL1 was upregulated 1.2-1.9-fold in sALS patients (Fig. 6B) compared to the mean of control samples by the standard curve method. $\triangle$ CT for PIWIL1 was reduced in sALS samples, which means an increase in RNA expression (Fig. 6A). The increase of PIWIL1 protein was confirmed by immunoblot (Fig. 6C, D). PIWIL4 was significantly downregulated an average of 0.6-fold in sALS patients (Fig. 6B) compared to control samples by the standard curve method. $\triangle$ CT for PIWIL4 was increased in SALS samples indicating a decrease in RNA expression (Fig. 6A). The decrease of PIWIL4 protein was also confirmed by immunoblot (Fig. 6C, D).

\section{PIWIL1- TDP-43 colocalization in postmortem samples}

We examined the expression pattern of PIWIL1 in the lumbar cord (L5) of SALS patients. In control cases, without neurodegenerative diseases, cell nuclei, nuclear membranes, and cytoplasm of anterior horn cells (AHCs) were positive for PIWIL1 (Fig. 7A). In the lumbar cord from sALS patients, AHCs were reduced in number and some remaining AHCs were atrophic with PIWIL 1-negative nuclei (Fig. 7B). In double immunofluorescence staining, PIWIL1 in cell bodies was partially co-localized with TDP-43-positive cytoplasmic inclusions in SALS patients (Fig. 7C-E).

\section{Discussion}

\section{piRNA dysregulation in SALS}

We have detected and validated dysregulation of five piRNAs in postmortem sALS samples. This is the first evidence of piRNA expression changes in brains of ALS patients, as well as the first report of these piRNAs in human brain. RT-qPCR validation of in silico piRNA target genes did not indicate significant alterations in their expression, suggesting that piRNAs have regulatory effects beyond in silico-predicted target coding gene regulation. Another possible interpretation of these piRNA alterations is that they are a byproduct or a manifestation of dysregulation in a different genomic region.

Generally, piRNAs contribute to silencing of transposable element (TE) expression, chromatin modifications [16], and mRNA localization [17]. The piRNA pathway targets a subset of LINE1 loci that contain full-length retrotransposon insertions [18], and there is growing evidence suggesting that PIWI proteins and piRNAs function mainly as transposable element regulators in the germline. Brains showing increased expression of retrotransposon elements are likely to be a source of altered piRNA production 
[19]. On the other hand, TDP-43 was reported to suppress transposable elements in ALS [20], and the loss of nuclear TDP-43 is associated with de-condensation of LINE retrotransposons [18]. The fact that all sporadic ALS samples used in this study manifested TDP-43 proteinopathy suggests that TDP-43 does not protect against TE, which may explain the dysregulation of piRNAs in postmortem medulla samples.

\section{PIWI protein dysregulation as a potential cause of ALS}

Our results demonstrate dysregulation in PIWIL1 and PIWIL4 in sALS samples. In humans, four PIWI proteins, PIWIL1-4, are involved in piRNA biogenesis and function. Expression of PIWI proteins differs by tissue and organ [21]. PIWIL1 regulates neuronal polarization and radial migration partly by modulating expression of microtubule-associated proteins (MAPs). MAPs are involved in axon extension by directly binding and stabilizing mRNA of some target genes, including MAP1B [22]. Previous observations show the protective effect of PIWIL1 in neuronal development and microtubule migration. Likewise, a recent study using postmortem samples of brains of Alzheimer's disease patients showed upregulation of PIWIL1[23]. Together these lines of evidence suggest that altered expression of PIWIL1 can be explained as a compensatory mechanism for axonal dysfunction. On the other hand, PIWIL1 was previously reported to interact physically with TDP-43 in Drosophila [24]. Immunohistochemistry results show PIWIL1 depletion in nuclei of sALS patients compared to controls. Moreover, colocalization of PIWIL1 and TDP-43 was also observed in the cytoplasm of sALS samples, suggesting that increased PIWIL1 and its interaction with TDP-43 in the cytoplasm may contribute to formation of TDP-43 inclusions. This also supports the idea that loss of function in the nucleus or compartmental mislocalization of PIWIL1 in neurons has a drastic impact on neurodegeneration in ALS.

In the current study, PIWIL2 expression was not detected in sALS postmortem samples, supporting the observation by Gasparini et al.[25]that PIWIL2 is expressed in neural stem/ progenitor cells, but not in neurons. PIWIL3 also was not detected by immunoblotting in these samples, while PIWIL4 was downregulated by $0.5-0.6$-fold in most sALS samples. PIWIL3 has not been reported previously in brain, as it has in ovary, testis, and blood [14]. The role of PIWIL4 in brain is poorly understood, despite recent reports indicating that PIWIL2 and PIWIL4 are associated with autism[26], and that PIWIL4 helps modulate neuronal differentiation from human embryonal carcinoma cells [27]. Disturbance of these tissue specificities of PIWI proteins, or dysfunction of PIWIL4 may also contribute to the disease signature of ALS.

Overall, our results suggest that dysregulation of the piRNA-PIWI protein axis is associated with the pathogenesis of ALS. PIWI protein mislocalization could be an important determinant of TDP-43 accumulation in the cytoplasm. Moreover, dysregulated piRNA and PIWI proteins may be useful as diagnostic biomarkers, as well as gene targets for molecular therapy of ALS.

\section{Conclusions}

This study is the first report on the involvement of PIWI/ piRNA metabolism in sALS brain tissues. Our results demonstrate significant dysregulation of five piRNAs, as well as PIWI proteins. They indicate that 
these piRNAs and PIWI proteins are involved in the pathogenesis of ALS, and may be useful as specific disease biomarkers. Furthermore, they may eventually serve as targets for development of novel therapeutics, such as nucleic acid medicines or gene therapeutics.

\section{Abbreviations}

ALS: Amyotrophic lateral sclerosis; fALS: Familial amyotrophic lateral sclerosis; sALS: Sporadic amyotrophic lateral sclerosis; ncRNA: Non-coding RNA; sRNA: Small RNA; miRNA: Micro RNA; piRNA: PIWI-interacting RNA; FDR: False discovery rate; PIWIL1: PIWI-like RNA-mediated gene silencing 1; PIWIL2: PIWI-like RNA-mediated gene silencing 2; PIWIL3: PIWI-like RNA-mediated gene silencing 3; PIWIL4: PIWIlike RNA-mediated gene silencing 4; RT-qPCR: Reverse transcription-quantitative PCR; TDP-43: TAR DNAbinding protein 43; AHC: Anterior horn cell, MAP: Microtubule-associated protein

\section{Declarations}

\section{Ethics approval and consent to participate}

Collection of autopsied human brain tissues and their use in this study were approved by the Ethics Committees of Osaka University Graduate School of Medicine, Tokyo Metropolitan Geriatric Hospital and Institute of Gerontology (TMGHIG) and the National Center of Neurology and Psychiatry (NCNP). Written informed consent was obtained from patient families. Experimental procedures involving human subjects were conducted in conformance with principles of the Declaration of Osaka University.

\section{Consent for publication}

Not applicable.

\section{Availability of data and materials}

Datasets generated and analyzed during the current study are available in the DNA Data Bank of Japan (DDBJ) under Temporary Submission ID: SSUB016807.

\section{Competing interests}

The authors declare that they have no competing interests.

\section{Funding}

This work was funded by the Grant-in-Aid for Scientific Research (C) (22590932, 25461302 and 16K09690 to SN), the Grant-in-Aid for Scientific Research on Innovative Areas (16H06277 to SN), AMED (JP20Im0203007 and JP20ek0109320 to SN, JP18dm0107103 to YS), grants from Japan Foundation for Neuroscience and Mental Health and Strategic Research Program for Brain Sciences (to SN). The 
funding bodies did not contribute to the design of the study, to collection, analysis, or interpretation of data, or to the writing of the manuscript.

\section{Authors' contributions}

RFA, SN, KO, GB, KI, KB, YN, YO and HM developed and organized the study. RFA, SN, KO, GB, ET, YY and JJ performed experiments. YS and SM collected and evaluated human samples. RFA and SN wrote the manuscript. All authors read and approved the final version of the manuscript.

\section{Acknowledgements}

This study was supported in part by Genome Information Research Center, Osaka University.

\section{References}

1. Mejzini R, Flynn LL, Pitout IL et al (2019) ALS Genetics, Mechanisms, and Therapeutics: Where Are We Now? Front Neurosci 13:. https://doi.org/10.3389/fnins.2019.01310

2. Prasad A, Bharathi V, Sivalingam V et al (2019) Molecular Mechanisms of TDP-43 Misfolding and Pathology in Amyotrophic Lateral Sclerosis. Front Mol Neurosci 12:. https://doi.org/10.3389/fnmol.2019.00025

3. Wakisaka KT, Imai Y (2019) The dawn of pirna research in various neuronal disorders. Front Biosci Landmark Ed 24:1440-1451

4. Mao Q, Fan L, Wang X et al (2019) Transcriptome-wide piRNA profiling in human brains for aging genetic factors. Jacobs J Genet 4

5. Martin M (2011) Cutadapt removes adapter sequences from high-throughput sequencing reads. EMBnetjournal 17:10. https://doi.org/10.14806/ej.17.1.200

6. Bolger AM, Lohse M, Usadel B (2014) Trimmomatic: a flexible trimmer for Illumina sequence data. Bioinforma Oxf Engl 30:2114-2120. https://doi.org/10.1093/bioinformatics/btu170

7. Kitchen R, exceRpt: The extra-cellular RNA processing toolkit. Includes software to preprocess, align, quantitate, and normalise smallRNA-seq datasets. https://rkitchen.github.io/exceRpt/

8. Waller R, Wyles M, Heath PR et al (2018) Small RNA Sequencing of Sporadic Amyotrophic Lateral Sclerosis Cerebrospinal Fluid Reveals Differentially Expressed miRNAs Related to Neural and Glial Activity. Front Neurosci 11:. https://doi.org/10.3389/fnins.2017.00731

9. Waller R, Goodall EF, Milo M et al (2017) Serum miRNAs miR-206, 143-3p and 374b-5p as potential biomarkers for amyotrophic lateral sclerosis (ALS). Neurobiol Aging 55:123-131. https://doi.org/10.1016/j.neurobiolaging.2017.03.027

10. Freischmidt A, Müller K, Ludolph AC, Weishaupt JH (2013) Systemic dysregulation of TDP-43 binding microRNAs in amyotrophic lateral sclerosis. Acta Neuropathol Commun 1:42. https://doi.org/10.1186/2051-5960-1-42 
11. Girard A, Sachidanandam R, Hannon GJ, Carmell MA (2006) A germline-specific class of small RNAs binds mammalian Piwi proteins. Nature 442:199-202. https://doi.org/10.1038/nature04917

12. Vagin VV, Sigova A, Li C et al (2006) A Distinct Small RNA Pathway Silences Selfish Genetic Elements in the Germline. Science. https://doi.org/10.1126/science.1129333

13. Piuco R, Galante PAF. piRNAdb. https://www.pirnadb.org/index

14. Wang J, Zhang P, Lu Y et al (2019) piRBase: a comprehensive database of piRNA sequences. Nucleic Acids Res 47:D175-D180. https://doi.org/10.1093/nar/gky1043

15. Nagano S, Jinno J, Abdelhamid RF et al (2020) TDP-43 transports ribosomal protein mRNA to regulate axonal local translation in neuronal axons. Acta Neuropathol (Berl) 140:695-713. https://doi.org/10.1007/s00401-020-02205-y

16. Yamanaka S, Siomi MC, Siomi H (2014) piRNA clusters and open chromatin structure. Mob DNA 5:22. https://doi.org/10.1186/1759-8753-5-22

17. Dufourt J, Bontonou G, Chartier A et al (2017) piRNAs and Aubergine cooperate with Wispy poly $(A)$ polymerase to stabilize mRNAs in the germ plasm. Nat Commun 8:1305. https://doi.org/10.1038/s41467-017-01431-5

18. Pezic D, Manakov SA, Sachidanandam R, Aravin AA (2014) piRNA pathway targets active LINE1 elements to establish the repressive H3K9me3 mark in germ cells. Genes Dev 28:1410-1428. https://doi.org/10.1101/gad.240895.114

19. Nandi S, Chandramohan D, Fioriti L et al (2016) Roles for small noncoding RNAs in silencing of retrotransposons in the mammalian brain. Proc Natl Acad Sci 113:12697-12702. https://doi.org/10.1073/pnas.1609287113

20. Tam OH, Ostrow LW, Gale Hammell M (2019) Diseases of the nERVous system: retrotransposon activity in neurodegenerative disease. Mob DNA 10:. https://doi.org/10.1186/s13100-019-0176-1

21. Rojas-Ríos P, Simonelig M (2018) piRNAs and PIWI proteins: regulators of gene expression in development and stem cells. Development 145:. https://doi.org/10.1242/dev.161786

22. Zhao P, Yao M, Chang $S$ et al (2015) Novel function of PIWIL1 in neuronal polarization and migration via regulation of microtubule-associated proteins. Mol Brain 8:39. https://doi.org/10.1186/s13041015-0131-0

23. Frost B, Hemberg M, Lewis J, Feany MB (2014) Tau promotes neurodegeneration through global chromatin relaxation. Nat Neurosci 17:357-366. https://doi.org/10.1038/nn.3639

24. Godena VK, Romano G, Romano M et al (2011) TDP-43 Regulates Drosophila Neuromuscular Junctions Growth by Modulating Futsch/MAP1B Levels and Synaptic Microtubules Organization. PLOS ONE 6:e17808. https://doi.org/10.1371/journal.pone.0017808

25. Gasperini C, Pelizzoli R, Van AL et al (2020) The piRNA pathway sustains adult neurogenesis by repressing protein synthesis

26. lossifov I, O'Roak BJ, Sanders SJ et al (2014) The contribution of de novo coding mutations to autism spectrum disorder. Nature 515:216-221. https://doi.org/10.1038/nature13908 
27. Subhramanyam CS, Cao Q, Wang C et al (2020) Role of PIWI-like 4 in modulating neuronal differentiation from human embryonal carcinoma cells. RNA Biol 17:1613-1624. https://doi.org/10.1080/15476286.2020.1757896

\section{Tables}

Table 1. Clinical characteristics of postmortem samples from sALS patients and controls.

Controls used in this study had no identified neurological disease.

\begin{tabular}{|c|c|c|c|}
\hline Control & Sex & Age & NPD \\
\hline 1 & $M$ & 68 & unremarkable \\
\hline 2 & $\mathrm{~F}$ & 73 & unremarkable \\
\hline 3 & $M$ & 81 & unremarkable \\
\hline 4 & $M$ & 76 & unremarkable \\
\hline 5 & $\mathrm{~F}$ & 80 & unremarkable \\
\hline 6 & $\mathrm{~F}$ & 84 & unremarkable \\
\hline 7 & $M$ & 57 & unremarkable \\
\hline ALS & Sex & Age & NPD \\
\hline 1 & $\mathrm{~F}$ & 83 & ALS、SDH \\
\hline 2 & $\mathrm{~F}$ & 75 & ALS \\
\hline 3 & $M$ & 77 & ALS \\
\hline 4 & $M$ & 79 & ALS \\
\hline 5 & $\mathrm{~F}$ & 56 & ALS \\
\hline 6 & $M$ & 67 & ALS \\
\hline 7 & M & 80 & ALS \\
\hline
\end{tabular}

M: male, F: female

NPD: neuropathological diagnosis

SDH: chronic subdural hematoma 
Table 2. miRNAs that were dysregulated in ALS samples. Two of them, hsa-miR-143-5p and hsa-miR-143$3 p$, have been previously reported as dysregulated in ALS [8-10].

\begin{tabular}{lc}
\hline miRNA & Q Value (FDR) \\
\hline hsa-miR-143-5p & $4.43 \times 10^{-2}$ \\
hsa-miR-143-3p & $1.42 \times 10^{-2}$ \\
hsa-miR-145-5p & $1.42 \times 10^{-1}$ \\
hsa-miR-27a & $1.42 \times 10^{-1}$ \\
hsa-miR-3607-3p & $1.42 \times 10^{-1}$ \\
hsa-miR-577 & $2.21 \times 10^{-1}$ \\
hsa-miR-122-5p & $2.21 \times 10^{-1}$ \\
hsa-miR-6847-5p & $2.21 \times 10^{-1}$ \\
hsa-miR-145-3p & $2.77 \times 10^{-1}$ \\
\hline
\end{tabular}

FDR: false discovery rate

Table 3. Differentially expressed piRNAs with FDR $<0.05$ and $P<0.05$.

\begin{tabular}{cc}
\hline piRNA & Q Value (FDR) \\
\hline hsa-piR-000578 & $4.65 \times 10^{-2}$ \\
hsa-piR-022184 & $4.65 \times 10^{-2}$ \\
hsa-piR-020871 & $1.44 \times 10^{-2}$ \\
hsa-piR-016735 & $4.65 \times 10^{-2}$ \\
hsa-piR-009294 & $4.65 \times 10^{-2}$ \\
\hline
\end{tabular}

FDR: false discovery rate

Table 4. Predicted target genes of dysregulated piRNAs, according to PiRBase and piRNAdb. In order to investigate changes in expression of in silico-predicted target genes for dysregulated piRNAs, we used 
piRBase and piRNAdb to predict target genes for validation. Most target genes were ribosomal protein pseudogenes, and 5S ribosomal RNA pseudogene transcript. Due to increased evidence of ribosomal protein dysregulation, we investigated expression of ribosomal protein coding genes, instead of pseudogenes.

\begin{tabular}{lll}
\hline piRNA & In silico predicted target gene & Validated gene \\
\hline hso-piR-000578 & RPL10P7 & RPL10 \\
hso-p R-022184 & RPL18AP3/TXNRD1 & RPL18A/ TXNRD1 \\
hso-piR-020871 & RPL13AP3 & RPL13A \\
hso-p R-016735 & RNA5SP202 & RNA5SP202 \\
hso-pR-009294 & RNA5-8SP6 & RNA5-8SP6 \\
\hline
\end{tabular}

Table 5. Primers used for qPCR validation of piRNAs and their predicted target genes 


\begin{tabular}{|c|c|}
\hline Target Gene & Sequence \\
\hline$\overline{p i R \_000578-F}$ & 5'-AGAACAAGGAGCATGTGATT-3' \\
\hline piR_022184-F & 5'-TACGAGAGTACAAGGTAGTGGG-3' \\
\hline piR_020871-F & 5'-AAAACTCTGAGGCTTTGGTGAG-3' \\
\hline piR_016735-F & 5'-CCGCCTGGGAATACCGGGTGCT-3' \\
\hline piR_009294-F & 5'-CATTGATCATCGACACTTCGAA-3' \\
\hline 3' Adaptor & 5'-rApp/CTGTAGGCACCATCAAT/3ddC-3' \\
\hline 3' Adaptor-RT & 5'-AAGGTCTATCAGCAGAAAAATTGATGGTGCCTACAG -3 \\
\hline \multicolumn{2}{|l|}{ U6RNA } \\
\hline U6RNA_qF & 5'-CTCGCTTCGGCAGCACATAT-3' \\
\hline U6RNA_qR & 5'-TATGGAACGCTTCACGAATTTG-3' \\
\hline Human PIWIL1 & variant $1 \& 2$ \\
\hline piwiL1_qF & 5'-GACCAGAATCCCAAGAGCACCT-3' \\
\hline piwiL1_qR & 5'-CTGACCAAGACAGGCTGCTTCA-3' \\
\hline Human PIWIL4 & variant 4 \\
\hline PIWIL4_qF & 5'-CTGTATCGGACCTGAATCAGCC-3' \\
\hline PIWIL4_qR & 5'-CAGATGTTGCCTGGTCAGTCAG-3' \\
\hline \multicolumn{2}{|l|}{ Human RPL10 } \\
\hline RPL10_qF & 5'-AAGGAGCATGTGATTGAGGCCCTG-3' \\
\hline RPL10_qR & 5'-ATGAGCCGTTTCTCAGCCACCATG-3' \\
\hline \multicolumn{2}{|l|}{ Human RPL13A } \\
\hline RPL13A_qF & 5'-GACTGGGAAGATGGGCAACAAAGG-3' \\
\hline RPL13A_qR & 5'-TGCCCTTCACTCTTTCTGCTCTAG-3' \\
\hline \multicolumn{2}{|l|}{ Human RPL18A } \\
\hline RPL18A_qF & 5'-CATGAAGGTGGAGGAGATCGCG-3' \\
\hline RPL18A_qR & 5'-GTGAAGCGTGGCTTGTGCTGAC-3' \\
\hline \multicolumn{2}{|l|}{ RNA5SP202 } \\
\hline RNA5SP202_qF & 5'-CTACGGCCATACCACCCTGAACG-3' \\
\hline RNA5SP202_qR & 5'-CTACAGCACCCGGTATTCCCAG-3' \\
\hline \multicolumn{2}{|l|}{ RNA5-8SP6 } \\
\hline RNA5-8SP6_qF & 5'-GATCACTCGGCTCCTGCGTTGATG-3' \\
\hline RNA5-8SP6_qR & 5'-GGCAAGCAAAGCTCAGACAGG-3' \\
\hline \multicolumn{2}{|l|}{ Human TXNRD1 } \\
\hline TXNRD1_qF & 5'-GTTACTTGGGCATCCCTGGTGA-3' \\
\hline TXNRD1_qR & 5'-CGCACTCCAAAGCGACATAGGA-3' \\
\hline \multicolumn{2}{|l|}{ Human GAPDH } \\
\hline GAPDH_qF & 5'-GTCTCCTCTGACTTCAACAGCG-3' \\
\hline GAPDH_qR & 5'-ACCACCCTGTTGCTGTAGCCAA-3' \\
\hline
\end{tabular}

\section{Figures}




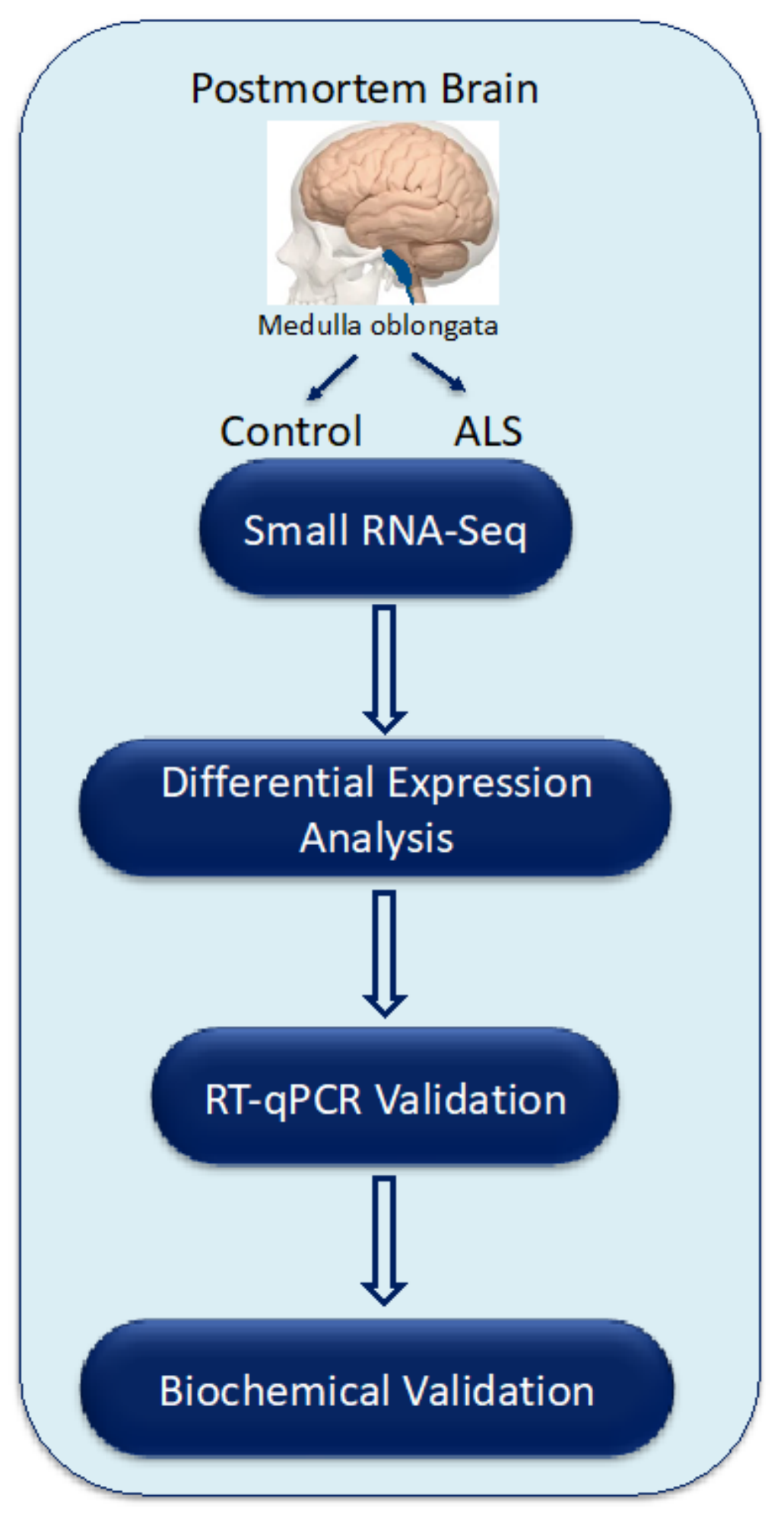

Figure 1

Schematic representation of the experimental design. Postmortem human tissue samples used in this study were collected from ALS cases $(n=7)$ and age- and sex-matched control cases $(n=7)$. RNA-Seq was performed on RNA extracted from the pyramidal tract of the medulla oblongata. Differential expression analysis of small RNAs was performed, followed by validation. 


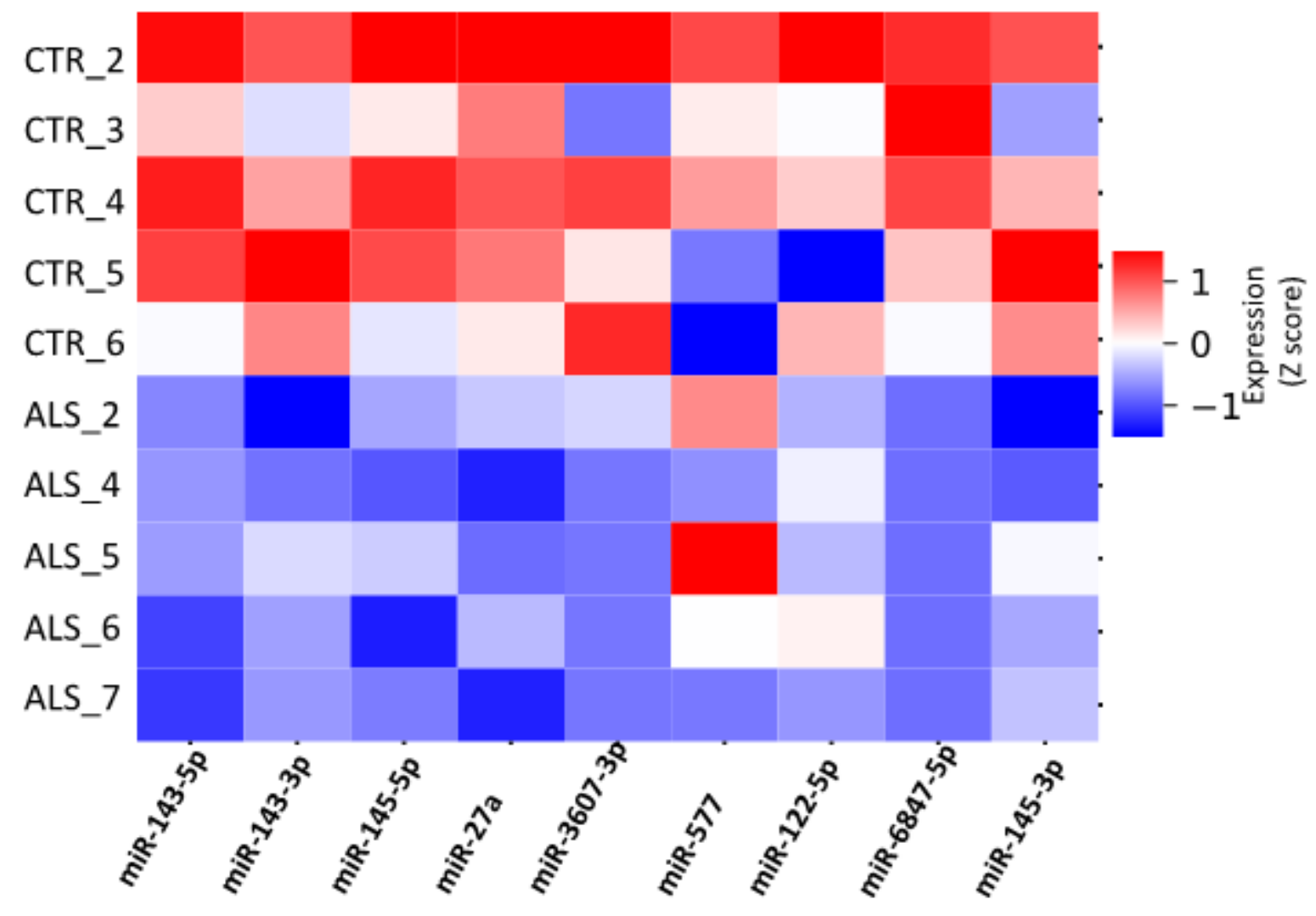

Figure 2

miRNAs are dysregulated in SALS. Differential expression analysis revealed that nine miRNAs were significantly dysregulated in ALS samples $(\mathrm{p}<0.05)$. Data were normalized as the log 10 read count per million (RPM). 


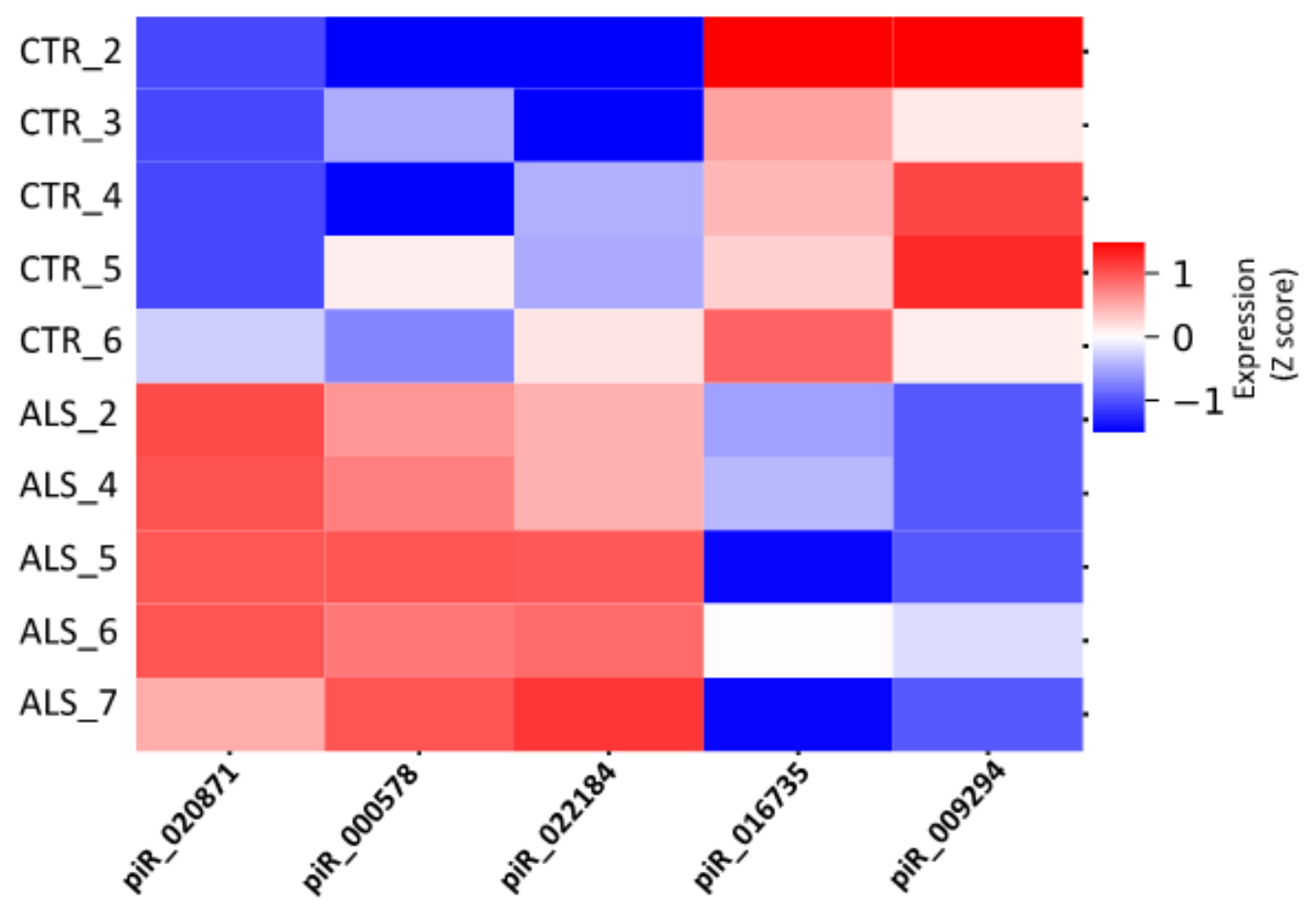

Figure 3

piRNAs are significantly dysregulated in ALS. Three piRNAs were up-regulated in ALS samples (red) and two were down-regulated (blue) $(p<0.05)$. Normalized as the log 10 read count per million (RPM). 
A
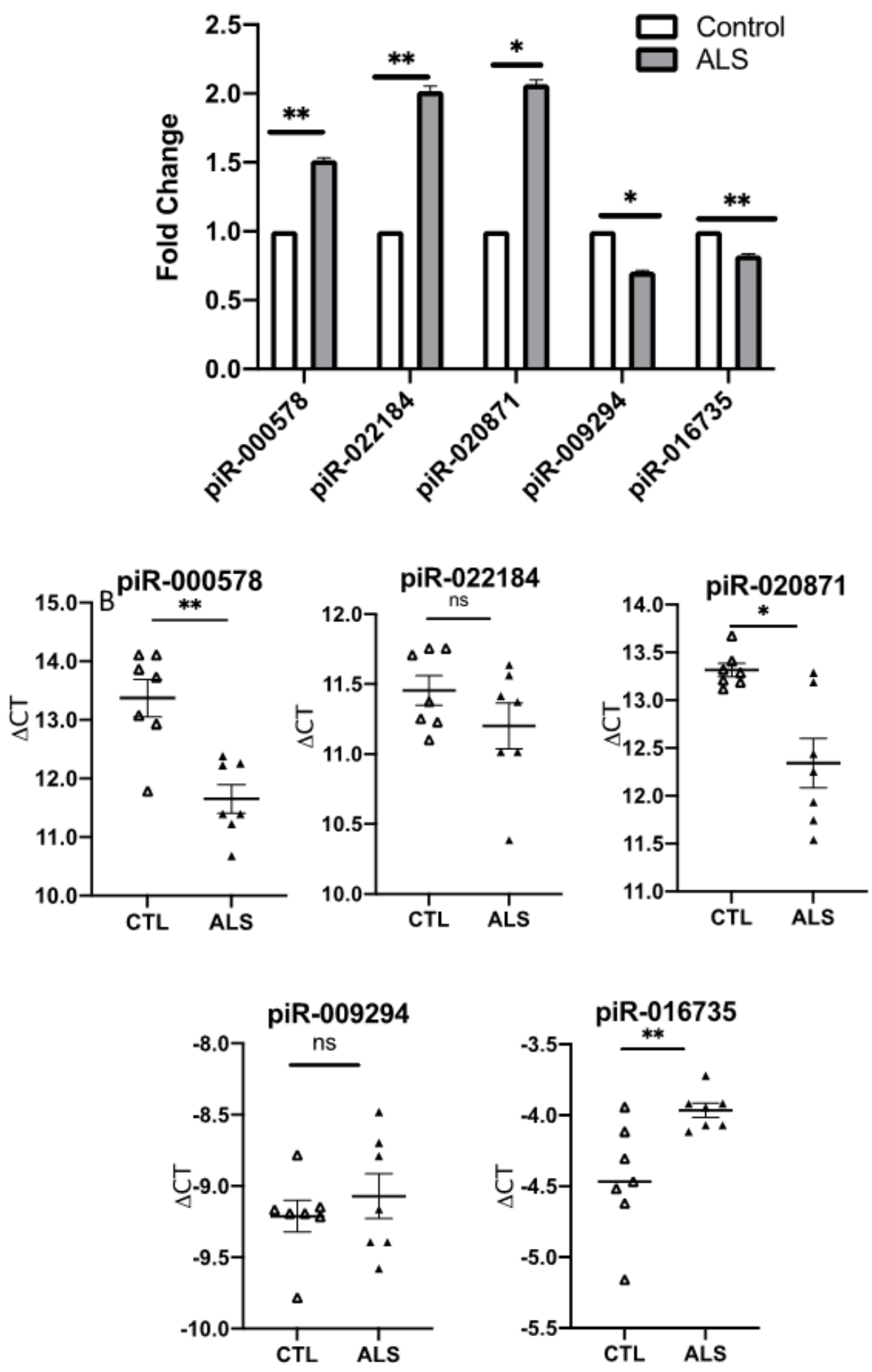

Figure 4

Validation of piRNA expression with RT-qPCR. Dysregulation of five piRNAs was validated using RT-qPCR with two methods, the standard curve method to visualize the overall trend for piRNA perturbation $(A)$ and the $\triangle C T$ method to quantify expression in individual samples $(B)$. Both methods confirm that these five piRNAs were significantly altered in SALS samples in comparison with controls. A. Average change in expression of each piRNA in control vs sALS samples. The Wilcoxon signed-rank test analysis was used 
for statistical calculations. Error bars denote SEMs. B. piRNA expression by $\triangle \mathrm{CT}$ in control vs SALS. The Mann-Whitney test was used for statistical calculations. ns: not significant. ${ }^{*} p<0.05,{ }^{\star *} p<0.01$.

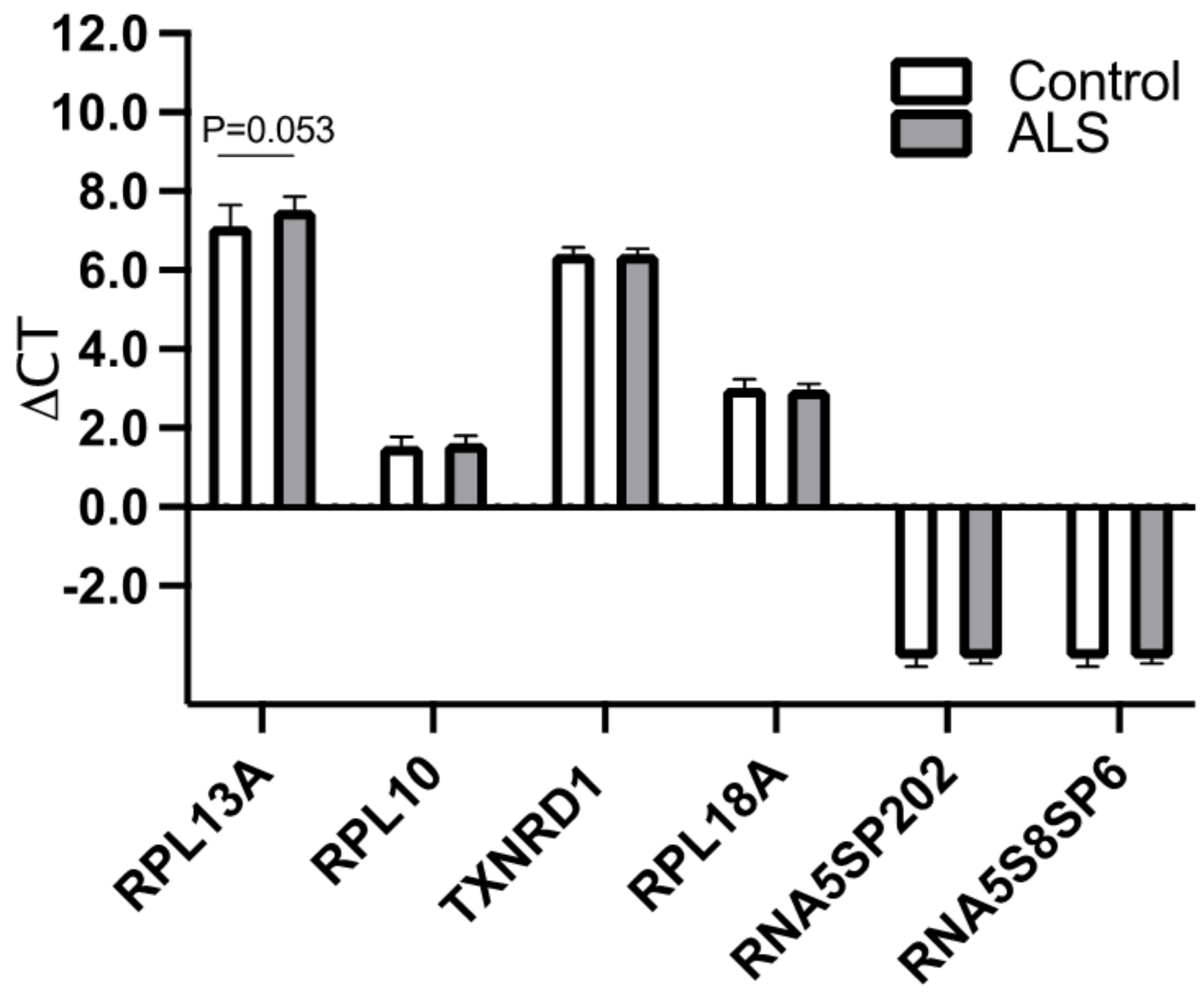

Figure 5

piRNA target genes are mostly ribosomal protein pseudogenes. The $\triangle \mathrm{CT}$ method was used to investigate changes in expression of in silico-predicted target genes for dysregulated piRNA, according to piRBase and piRNAdb (Table 4). Target genes included ribosomal protein pseudogenes, RPL10P7, RPL13AP3, RPL18AP3, and 5S ribosomal RNA pseudogene transcripts, RNA5SP202 and RNA5-8SP6. There were no notable changes except for expression of RPL13A ( $p=0.053)$. Among expected target genes, no significant difference of TXNRD1 was observed between SALS and control samples. This suggests that these piRNAs have regulatory effects beyond regulation of in silico-predicted target coding genes. 
A

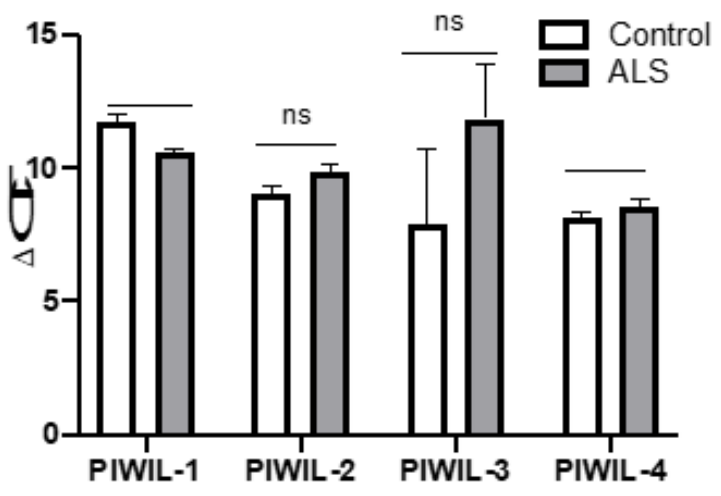

B
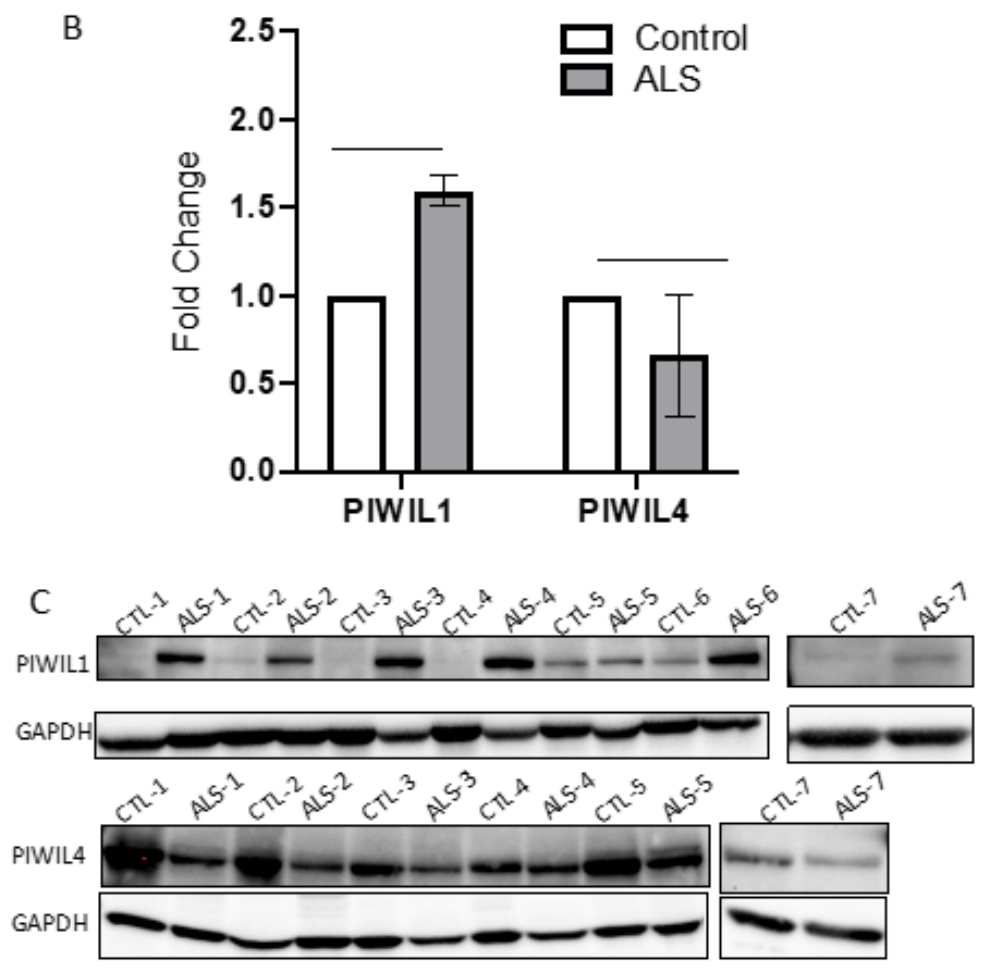

D
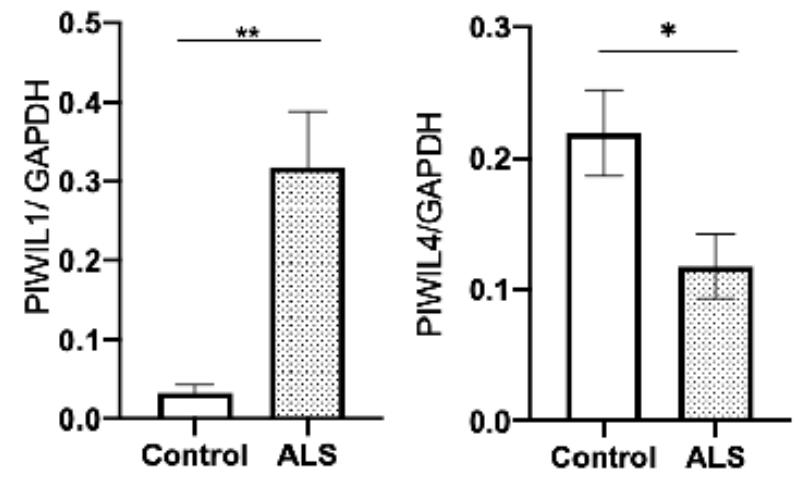

Figure 6

PIWI protein was dysregulated in postmortem sALS samples. A. RT-qPCR for PIWIL1, PIWIL2, PIWIL3, PIWIL4 expression of mRNA using the $\triangle C T$ method. RT-qPCR using the $\triangle \mathrm{CT}$ method for PIWIL1 and PIWIL4 showed significant differences between ALS and control samples, while PIWIL2 and PIWIL3 showed insignificant differences. B. RT-qPCR results of postmortem PIWIL1 and PIWIL4 expression using a control pool for the standard curve method. A one-tailed t-test and the Wilcoxon test were used for 
statistical calculations. PIWIL1 was upregulated 1.2-1.9-fold in SALS patients compared to the mean of control samples, by the standard curve method. C. Western blot analysis of PIWIL1 and PIWIL4 in postmortem samples. The increase of PIWIL1 protein and the decrease of PIWIL4 protein were confirmed in ALS samples. D. Quantitative analysis of PIWIL1 and PIWIL4 in a Western blot normalized to GAPDH. The increase of PIWIL1 protein and the decrease of PIWIL4 protein were statistically confirmed. ns: not significant. ${ }^{*} p<0.05,{ }^{*} \mathrm{p}<0.01$.
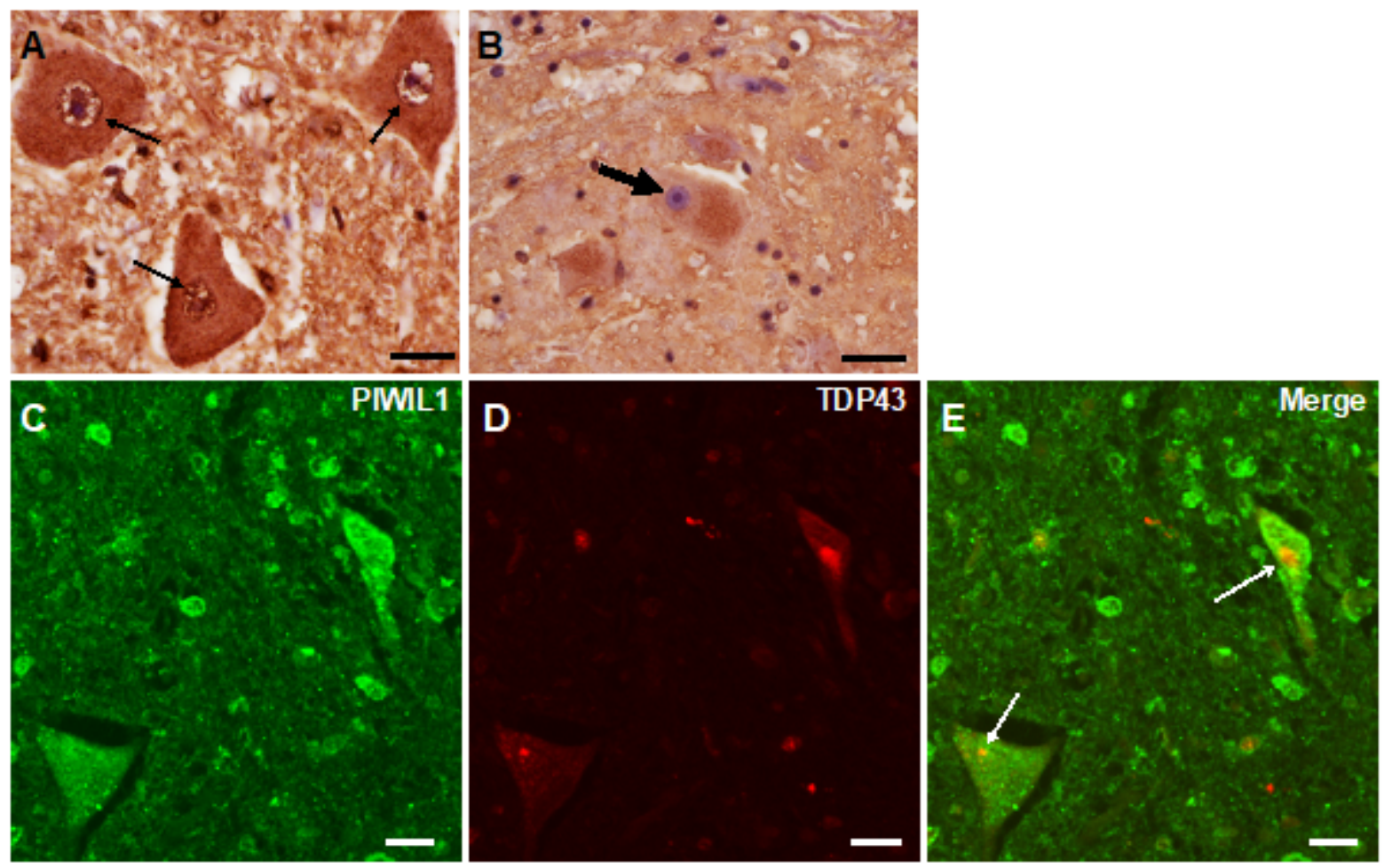

Figure 7

Immunohistochemistry for PIWIL1 in the lumbar spinal cord of control (A) and ALS (B-E) patients. (A) In control patients, cell nuclei, the nuclear membrane (small arrows), and cell bodies of anterior horn cells (AHCs) are immunopositive for PIWIL1. (B) In ALS patients, some AHCs have nuclei negative for PIWIL1 (arrow). (C-E) In double immunofluorescence staining, PIWIL1 is co-localized with intracytoplasmic inclusions positive for TDP-43 (white arrows) in ALS patients. Scale bar $=20 \mu \mathrm{m}$.

\section{Supplementary Files}

This is a list of supplementary files associated with this preprint. Click to download.

- Supplementarytable20210823.docx 